\title{
Measurement and therapeutical system based on Universal Serial Bus
}

\author{
Lukas Palko ${ }^{1}$ \\ Department of Telecommunications \\ Faculty of Electrical Engineering and Communication \\ Brno University of Technology, Czech Republic ${ }^{1}$ \\ lucas.palko@enjoy-rampl.com
}

\begin{abstract}
The paper presents complex treatment and measurement system based on USB and its enhancements. The article is divided into two parts. The first one deals with up-to-date USB enhancements which are USB On-The-Go, USB Test \& Measurement Class, Wireless USB, PictBridge, PoweredUSB, Inter-Chip USB, Battery charging specification. The second part presents the practical usage for measurement and therapeutical system.
\end{abstract}

Keywords: USB, USB On-The-Go, OTG, USB Test \& Measurement Class, USBTMC, Wireless USB, WUSB, PictBridge, PoweredUSB, Retail USB, USB PlusPower, USB +Power, Inter-Chip USB, Battery charging specification.

\section{Introduction}

The theme of this paper is to find the communication solution for the research into the possibility of skin therapy by the digitized form of magnetotherapy and phototherapy.

The digitization approach enables an easy control of batching exactly determined amounts of magnetic and electromagnetic (luminous) energy in space and time into the treated environment as well as the transmission of necessary control and diagnostic information between the therapist and the patient. Developing this method has had impacts that were not quite anticipated. There is supposed a controlled treatment of damaged and otherwise degraded cells of skin (burns, bumps, infection, etc.).

In view of the known contraindications in both magnetotherapy and phototherapy (laserotherapy), interest has concentrated on the joint action of planar pulsed magnetic and luminous fields penetrating into small depths of human skin. This medical technology is based on generating a combination of the two types of field close above the skin, according to a computation algorithm given in advance.

Here are the research aims:

- development of a laboratory type of therapeutic device that would allow research into digitally controlled planar pulsed magnetic and luminous fields,

- studying the effect of this combined field on biological materials, especially on human skin, with simultaneous search for suitable chemotherapeutics and their application possibilities, 
- preparation of a user-friendly form of the controlling device software, inclusive of keeping records of therapist's patients included in the experimental program,

- complementing the therapeutic device with an imaging equipment, with the possibility of the therapist demarcating the surface under treatment directly on the computer display, controlling the device from a distance to facilitate an interactive out-patient treatment as registered in the program.

The measurement and therapeutical system contains a stand-alone therapeutic device for dermatology and cosmetics (one of the research aims), medical diagnostics apparatuses (ultrasound scanner, skin surface roughness diagnostics, skin parameters analyzer - amount of sebum, hydration, skin elasticity, water loss survey, skin conductivity) and additional utilities for better patient monitoring (PDA, digital camera). The whole system is intended to find out effects of the dermatology therapeutic device.

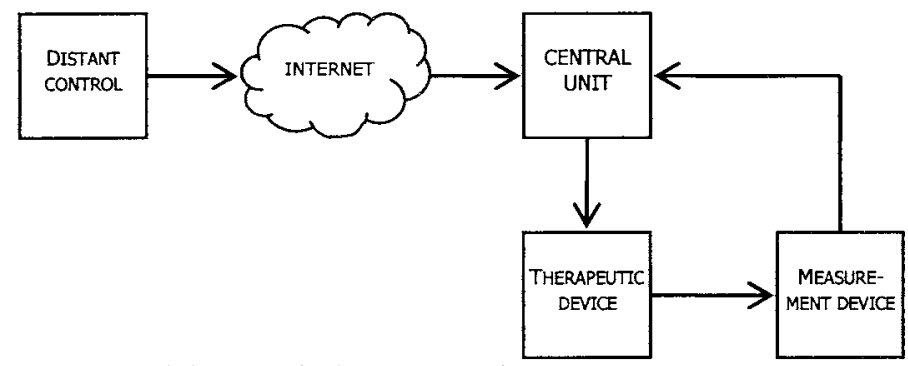

Fig. 1. Measurement and therapeutical system architecture

As the most perspective communication interface was chosen USB with its enhancements. Next chapter gives a brief survey of USB basics and its enhancements, which are supposed to use in the project.

\section{USB enhancements}

The very first idea of USB was to create uniform interface for different computer peripheral units. This point was completed and even more it was broken through. The USB become the most popular PC interface and it practically pushed away other interfaces. This huge popularity is given by unique combination of proprieties:

- plug-and-play feature - user friendly, no troubles with plugging or unplugging, can be connected to a PC "on-the-fly" without need for restart,

- high transfer rates up to $480 \mathrm{Mbps}$ - sufficient for most of applications,

- supply "on board" - VBUS contains DC $+5 \mathrm{~V}$ power source with current drain up to $500 \mathrm{~mA}$,

- plenty of connected devices .... to one host can be connected up to 127 peripherals,

- serial communication - the count of wires was reduced down to 4 (including supply), the cables are easy to manipulate with.

It is obvious that all of these features are not exactly the best, but the combination seems to be excellently balanced. There exists a FireWire Standard, which offers 
higher transfer speed and it is used for multimedia signal transfers, but it legs behind the USB in other features. Here are shown the USB enhancements which push this phenomenon beyond the state of art [1], [2].

\subsection{USB On-The-Go (OTG)}

The USB OTG was created for making the current USB interface available for smaller portable devices (PDA, digital camera, mobile phone, MP3 player, etc.). A basic goal is apart from smaller connectors an ability to mutually communicate oneto-one without master computer. USB OTG became very important standard for uniforming different manufacture approaches and it guarantees compatibility across platforms. The interface independency offers for example a contacts synchronization of a mobile phone and a PDA, a connection between GPS and PDA, a song exchange between two MP3 players, etc. Implementation of existing USB interface offers a wide bandwidth and so a transfer rate up to $480 \mathrm{Mbps}$. This high rate allows mass data transfer.

The classic USB uses architecture of a master and a slave. A USB host (PC) acts as the master and a USB peripheral (USB Device) acts as the slave. Only the USB host can control the configuration and data transfers over the link and initiate data transfers. Against that is USB OTG, which brings new possibility. The USB-OTG device is able to initiate the session, control the connection and exchange host and peripheral roles between each other. There are two special protocols:

- Session Request Protocol (SRP) allows both devices (host and peripheral) to enable or disable the active status of the communication link. It is very important for power consumption control of portable devices.

- Host Negotiation Protocol (HNP) brings possibility to exchange the host and the peripheral roles. Devices, which have implemented HNP, are called OTG dual-role devices. Here is point, that both communicating devices can initiate the data transfer.

The USB OTG is backward compatible with common USB. When the OTG device is connected to USB (no OTG), it acts as a common USB host or peripheral. The same effect of loosing OTG capabilities will arose after connection of USB hub, all OTG devices will become common USB devices (one of these will be a host a the others will be peripherals) [1], [3], [8].

\subsection{Wireless USB (WUSB)}

The basic idea of WUSB is to combine the speed and user-friendly technology with the wireless comfort. The range is supposed to the short-range category alike a Bluetooth technology. It is designed as a substitution for all devices that are now connected by common USB (printers, scanners, MP3 players, hard discs, game controllers, etc.). It used the WiMedia Alliance's Ultra-WideBand (UWB) common radio platform which offers transmission rates up to $480 \mathrm{Mbps}$ at 3 meters and up to $110 \mathrm{Mbps}$ at 10 meters.

The radio operates between 3.1 and $10.6 \mathrm{GHz}$ and it can be adjusted according to a local regulatory policy in different countries around the world. A maximal count of 
devices remains on 127 , but there is no longer need for hubs - all devices are connected directly to the host. There became new types of adapters:

- Device Wire Adapter (DWA) for existing USB devices to connect to the wireless host,

- Host Wire Adapter (HWA) for personal computer to gain a wireless host capability. It can be connected externally to desktop common USB ports or to Card-Reader of laptop.

Apart from that, there is one additional feature - Dual-Role Devices (DRDs) support. It is an approach to a USB-OTG with limited capabilities. There is given a chance for a typical device to become a host for specified activity. For example a digital camera is device for a desktop but even more it can control a direct image transfer to a printer $[3],[5],[8]$.

\subsection{USB Test \& Measurement Class (USBTMC)}

This class is intended for automated measurement systems to interconnect a central control unit (usual computer) and measurement devices. It becomes a modern alternative to current interfaces GPIB (IEEE-488) and RS-232. The USBTMC development is based on Virtual Instrument Software Architecture (VISA), which is supported by GPIB and also RS-232. The USBTMC is a result of a collective effort of companies joined in VISA and USB Implementers Forum (USB IF) community.

USBTMC is able to control signals of measurement devices and accept their requests for interruption as well. Physical layer of USBTMC is equal to classic USB, thus the user comfort remains with all advantages:

- number of connected devices up to 127 ,

- high speed up to $480 \mathrm{Mbps}$,

- plug-and-play feature.

USBTMC can be used as a GPIB-USB converter. It enables to connect older GPIB measurement instruments to common PC via the USB. A bit rate is adapted to GPIB capabilities and it can be up to $8 \mathrm{Mbps}$ (or $64 \mathrm{Mbps}$ for an extension version). The converter acts for an operating system as a virtual GPIB port and therefore there are no additional software needs. Current GPIB measurement software detects a device as a standard GPIB [1], [2].

\subsection{Inter-Chip USB}

The Inter-Chip USB (IC USB) is defined in a supplement to the USB 2.0 specifications for inter chip communication up to $10 \mathrm{~cm}$ distance. IC_USB specifications define a USB physical layer adapted for embedded devices. The main change to a USB standard is definition of multiple operating voltage classes $(1.0 \mathrm{~V}$, $1.2 \mathrm{~V}, 1.5 \mathrm{~V}, 1.8 \mathrm{~V}$ and $3.0 \mathrm{~V}$ ) which facilitate migration to planned higher-density IC technology. The features are:

- all IC_USB products (host, peripheral) are USB products able to use USB compliant software,

- any peripheral device may by supplied by one of power-supply voltage selected from mentioned voltage classes, 
- Full-speed (12 Mbps) support is required for all IC_USB products, Low-speed

(1.5 Mbps) support is required only for hosts, for peripherals it is optional and High-speed (480 Mbps) is reserved for future study.

This specification is supported by all of Europe's major mobile network operators and it will be probably adopted by Multi Media Card Association (MMCA) into a MMC I/O specification. Another usage is for microcontroller technology [3], [7].

\subsection{PictBridge}

PictBridge is a standard related to the USB for direct printing. It was developed by Camera \& Imaging Products Association (CIPA) and the aim was to print images directly from a digital camera (DSC) to a printer without a need for additional control equipment (computer). It is an open standard, but it cannot be published or revealed to the third party without a permission of CIPA. Printers have usually USB port type A and portable devices have usually USB port type Mini-B. There is no need for additional drivers and control software and all settings depend on devices firmware. PictBridge offers these features:

- printing one image displayed on the DSC or determined selection of more then one,

- automatic printing of images using the Digital Print Order format (DPOF) specification.

PictBridge is expanded standard and it is supported by most of producers of digital cameras, camcorders and also printers [6], [8].

\subsection{PoweredUSB}

The USB has it own supply distribution (VBUS), where is available voltage $+5 \mathrm{~V} \mathrm{DC}$ and current up to $100 \mathrm{~mA}$ (500 mA after announcement) for each USB-hub port. It can be sufficient for smaller devices like mice, keyboards, web cams, etc., but when the requirements exceed this limitation, there is a need for external power source and the advantage of real plug-and-play is devalued.

The PoweredUSB (also know as Retail USB, USB PlusPower, USB +Power) brings extra power lines to the common USB, which can fulfill the higher requirements and it also remains full compatibility to common USB. There became a new robust connector with 4 additional pins. This standard was developed by IBM, NCR, and FCI/Berg.

There are three key position locations on host connector. It determines which of these voltages is available in the connector:

- $\quad+5 \mathrm{~V} \mathrm{DC}$, amperage up to $6 \mathrm{~A}$ (power up to $30 \mathrm{~W}$ ),

- $\quad+12 \mathrm{~V} \mathrm{DC}$, amperage up to $6 \mathrm{~A}$ (power up to $72 \mathrm{~W}$ ),

- $\quad+24 \mathrm{~V} \mathrm{DC}$, amperage up to $6 \mathrm{~A}$ (power up to $144 \mathrm{~W}$ ).

There is special requirement for the used cable, because of need for high transmitting rate and capability to supply up to $6 \mathrm{~A}$ on mentioned voltages. The recommended maximum length is 4 meters. Hot-Plugging (Hot-Unplugging) feature known from 
USB is supported by a construction of connectors, where ground pins are front-ended the voltage ones. This feature partially suppresses contact arcing into the connector, in spite of it, the devices should be designed to avoid connector degradation or electronic component failure that could occur [4].

\subsection{Battery charging specification}

This specification was developed by the USB Implementers Forum Battery Charging workgroup for defining a standard way to draw electrical current from PCs. In addition, it allows PCs to control levels of current, in order to shorten the charging time of connected portable devices. The USB port is currently used for charging batteries in peripheral devices without any closer description and the trend among mobile devices to utilize USB for charging is growing. The new specification allows multiple types of portable devices to charge from USB ports thus it eliminates the need to carry multiple chargers for different kinds of devices.

The main benefit is definition of mechanism for portable devices of power source detection. If the source is wall charger or high current host or high current hub, the system allows immediately draw currents transcending $500 \mathrm{~mA}$ barrier. If the source is $\mathrm{PC}$ or hub, peripheral devices are limited up to $100 \mathrm{~mA}$ or $500 \mathrm{~mA}$ according to USB specification. The group also comes up with a provision in the new specs to allow portable devices with dead batteries to recharge at $100 \mathrm{~mA}$ even if they are not able to power up [3].

\section{Application}

All of these additions to the USB are intended to use in measurement and therapeutical system: 


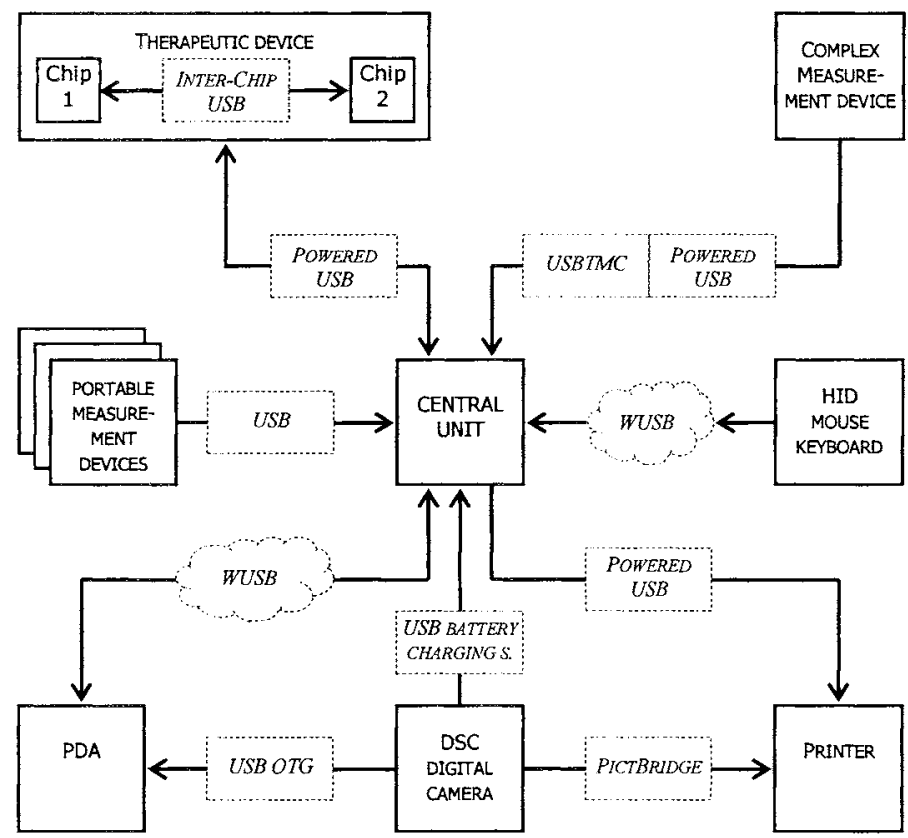

Fig. 2. Interface block diagram of the measurement and therapeutical system.

The core of the system is the central unit which is implemented into a high performance personal computer. It is operated wirelessly by WUSB keyboard and mouse and it has these key functions:

- controlling of the therapeutic device with a safety feedback,

- acquisition and processing of measured data,

- internet connection to a distant control center (not shown on the figure 2).

The therapeutic device is connected to the central unit via the PoweredUSB, which ensures both power supply and data link. Data link is used for the device control with a safety feedback. The power supply need is fulfilled by voltage $+12 \mathrm{~V}$.

Inside the device are placed integrated microcontrollers. Their data exchange is via Inter-Chip USB specification.A complex measurement device is a GPIB diagnostic apparatus, which uses USBTMC transducer, for a measurement data transfer simplification. Power supply is provided by the PoweredUSB link with $+24 \mathrm{~V}$.

A block mentioned as portable measurement devices means a set of simple medical diagnostic apparatuses for skin parameters measurement. Most of them are equipped by the common USB and the rest has a RS-232 output. These are supplemented by converters to the USB. PDA and a digital camera (DSC) are used for better patient monitoring out of the central control unit. The PDA uses wireless connection (WUSB) to the central unit. The DSC can be either connected to the PDA by the USB on-to-go or directly to the central unit, when it can use the USB battery charging specification for both images downloading and a battery charging. The last is an optional printer which can be powered by the PoweredUSB from the central unit and it can be used for a direct images printing from the DSC with the aid of the PictBridge enhancement. 


\section{Conclusions}

There were designed three generations of the therapeutic device for dermatology and cosmetics. The block diagram of the actual measurement and therapeutical system with the third generation device is showed on the figure 2. Among the features of the third generation therapeutic device belongs a simultaneous planar pulsed magnetic and luminous field generating, dynamic intensity field application, a surface that is acted on demarcation and application visualization on the computer display for the operator to have an idea of its dynamic course.

The USB as a communication interface was found suitable for the system by virtue of its versatility and easy of use in all enhancements.

The results, obtained during the research into the possibility of skin therapy by the digitized magnetotherapy and phototherapy, were presented at the first international conference on the Digital Society ICDS 2007 in Guadeloupe, French Caribbean [9].

\section{References}

1. Palko, L.: Modern trends of serial interface USB (in Czech). Article in electrotechnic magazine Elektrorevue, 2005, ISSN 1213-1539, article number 05025,

2. Palko, L.: Universal Serial Bus (in Czech). Article in technical magazine Automa (12/7), 2006, ISSN 1210-9592,

3. USB Implementers Forum. [citation 2007-05-13]. Available on http://www.usb.org

4. PoweredUSB.org, [citation 2007-05-13]. Available on http://www.poweredusb.org

5. Cypress webpage, [citation 2007-05-22]. Available on http://www.cypress.com

6. PictBridge website, (Camera \& Imaging Products Association) [citation 2007-05-23]. Available on http://www.cipa.jp/pictbridge

7. Léonard, Y.: MMC on the verge of adopting USB. [citation 2007-05-23]. Available on http://www.smartcardstrends.com

8. Wikipedia, the Free Encyclopedia. [citation 2007-05-13]. Available on http://www.wikipedia.org

9. Resl, V., Leba, M., Lojek, A., Číž, M., Hyršl, P., Palko, L., Rampl, I.,: Digitized biophysical therapy of skin. ICDS 2007 [including workshop TELEMED 2007]. Guadeloupe, French Caribbean, 2007, ISBN 0-7695-2760-42007 